\title{
An adaptive direct multisearch method for black-box multi-objective optimization
}

\author{
Sander Dedoncker · Wim Desmet • Frank Naets
}

the date of receipt and acceptance should be inserted later

\begin{abstract}
At present, black-box and simulation-based optimization problems with multiple objective functions are becoming increasingly common in the engineering context. In many cases, the functional relationships that define the objective and constraints are only known as blackboxes, cannot be differentiated accurately, and may be subject to unexpected failures. Directional direct search techniques, in particular the direct multisearch (DMS) methodology, may be applied to identify Pareto fronts for such problems. In this work, we propose a mechanism for adaptively selecting search directions in the DMS framework, with the goal of reducing the number of black-box evaluations required during the optimization. Our method relies on the concept of simplex derivatives in order to define search directions that are optimal for a local, linear model of the objective function. We provide a detailed description of the resulting algorithm and offer several practical recommendations for efficiently solving the associated subproblems. The overall performance in an academic context is assessed via a standard benchmark. Through a realistic case study, involving the bi-objective design optimization of a mechatronic quarter-car suspension, the performance of the novel method in a multidisciplinary engineering setting is tested. The results show that our method is competitive with standard implementations of DMS and other state-of-the-art multi-objective direct search methods.
\end{abstract}

Keywords derivative-free optimization · multi-objective optimization · bound-constrained optimization $\cdot$ direct multisearch $\cdot$ simplex derivatives

\section{Introduction}

Algorithms for derivative-free optimization (DFO) (Conn et al. 2009, Audet and Hare 2017) remain important tools for most quantitative disciplines. In many real-world optimization problems, common mathematical assumptions such as differentiability are not valid or derivatives may be computationally infeasible to obtain. In particular, the widespread adoption of complex

Correspondence: Sander Dedoncker

KU Leuven, Department of Mechanical Engineering Celestijnenlaan 300 box 2420 B-3001 Leuven (BE)

E-mail: sander.dedoncker@kuleuven.be

Sander Dedoncker [0] - Wim Desmet · Frank Naets

KU Leuven, Department of Mechanical Engineering

Flanders Make, DMMS Core Lab 
simulation software is confronting designers with objectives and constraints that are often impossible to analyze in detail, may not always produce meaningful values (e.g. when code errors occur) and may suffer from severe (numerical) noise. Applying derivative-free and black-box optimization algorithms is typically the only option in such cases.

In addition, it can be difficult to treat practical optimization problems within the usual framework of minimizing a single scalar cost function subject to multiple hard mathematical constraints. Multi-objective optimization (MOO) problems, which require the simultaneous optimization of several (conflicting) objectives, are frequently encountered in engineering and other technical fields. As opposed to single-objective optimization (SOO), solutions are not given as optimal points but rather as descriptions of the trade-offs that exist between the different objectives (Miettinen 1998).

As such, the preferences of the decision maker play an important role in qualifying solutions. MOO algorithms are generally classified based on the moment these preferences are taken into account (Marler and Arora 2004). A first category of methods relies on a priori articulation of preferences: before the optimization procedure starts, the objectives are aggregated in some desirable way. This procedure effectively converts the original problem to the single-objective case. In contrast, techniques employing a posteriori articulation of preferences aim to establish an approximation of the entire Pareto front. This way, the decision maker can assess which trade-offs are possible for the problem at hand.

The subject of the present paper is to develop a derivative-free, a posteriori algorithm for solving constrained MOO problems of the general form

$$
\begin{array}{ll}
\min _{\mathbf{x}} & \mathbf{f}(\mathbf{x}) \\
\text { s.t. } & \mathbf{x} \in \Omega,
\end{array}
$$

where $\mathbf{f}: \mathbb{R}^{n} \rightarrow \mathbb{R}^{p}$ is the (vector-valued) objective function, $\mathbf{x} \in \mathbb{R}^{n}$ is the design vector, and $\Omega \subset \mathbb{R}^{n}$ represents the feasible set. We focus in particular on the bound-constrained case, so

$$
\Omega=\left\{\mathbf{x} \mid \mathbf{b}_{l} \leq \mathbf{x} \leq \mathbf{b}_{u}\right\} .
$$

Here, $\mathbf{b}_{l}$ and $\mathbf{b}_{u}$ are vectors providing element-wise lower and upper bounds on the design vector. Considering black-box and simulation-based optimization (BBO, SBO) in engineering, the bound-constrained formulation (1) - (2) already covers a substantial amount of interesting applications. Since the design variables often represent physical quantities, truly unconstrained problems are quite rare in practice. Of course, more involved restrictions on the design variables are typically also present, but are often difficult to state explicitly with a constraint function. Therefore such constraints are assumed to be implicit in an extended-valued objective function $\mathbf{f}: \mathbb{R}^{n} \rightarrow \mathbb{R}^{p} \cup\{+\infty\}^{p}$.

Derivative-free, a posteriori methods have seen considerable advances over the past years. Many algorithms originally developed for the single-objective case have been adapted to the multi-objective setting. Biology- and physics-inspired metaheuristics were among the first to be generalized in this manner - see Coello Coello et al. (2007) for an extensive overview. Some examples are genetic algorithms (Deb et al.||2002), simulated annealing (Suppapitnarm et al. 2000) and particle swarm optimization (Coello Coello and Lechuga 2002). More recent research has focused on the extension of several deterministic derivative-free methods. Perhaps the most straightforward approach is to use scalarization, where the multi-objective problem is treated as a family of single-objective problems to which conventional optimization methods can be applied. Examples can be found in the works of Audet et al. (2010, 2008); Zapotecas-Martínez 
and Coello Coello (2016); Ryu and Kim (2014). Methods that are more 'native' to the multiobjective situation - in the sense that the objective function is never aggregated - have also been developed. The algorithms DFMO (Liuzzi et al.|2016), MODIR (Campana et al. 2018) and MOIF (Cocchi et al. 2018) can be classified here. In the present work, we introduce a method that is an instance of direct multisearch (DMS) (Custódio et al. 2011) and therefore also a member of this latter group.

Our work is further organized as follows. Section 2 explains some notational conventions and preliminary definitions, mainly intended for the reader who is unfamiliar with MOO. In Section 3, the general DMS framework is briefly recalled. Section 4 re-introduces the concept of simplex derivatives, in particular Jacobian matrices, for multi-objective functions. Taking an approach similar to GIGS (Dedoncker et al. 2021), we then propose a strategy for combining these concepts in an adaptive DMS instance. Our method, dubbed steepest-descent direct multisearch (SD-DMS), is described in detail in Section 5. Next, some important implementation aspects for constructing a practical algorithm are highlighted. We document numerical experiments on an academic benchmark in Section 7 and study an engineering case in Section 8 , Section 9 concludes the text.

\section{Preliminaries on multi-objective optimization}

We adopt the following notational convention with regards to vectors $\mathbf{v}, \mathbf{w} \in \mathbb{R}^{n}$ :

$$
\begin{array}{ll}
\mathbf{v} \leq \mathbf{w} \Longleftrightarrow v^{(i)} \leq w^{(i)} & \forall i \in\{0,1, \ldots, n\}, \\
\mathbf{v}<\mathbf{w} \Longleftrightarrow v^{(i)}<w^{(i)} & \forall i \in\{0,1, \ldots, n\},
\end{array}
$$

in other words, vector inequalities are interpreted element-wise. This partial order is used to evaluate the output of the vector-valued objective function $\mathbf{f}$. Given two design points $\mathbf{x}, \mathbf{y} \in \mathbb{R}^{n}$, we say $\mathbf{x}$ dominates $\mathbf{y}(\mathbf{x} \prec \mathbf{y})$ according to the relation:

$$
\mathbf{x} \prec \mathbf{y} \Longleftrightarrow \mathbf{f}(\mathbf{x}) \leq \mathbf{f}(\mathbf{y}), \mathbf{f}(\mathbf{x}) \neq \mathbf{f}(\mathbf{y}) .
$$

If neither point dominates the other, the two points are said to be nondominated or indifferent.

The concept of dominance makes it possible to establish design ranking and a notion of optimality in the multi-objective sense: a design $\mathrm{x} \in \Omega$ is Pareto optimal for the problem (1) if no feasible $\mathbf{y}$ dominates it. Generally speaking, the different scalar objectives impose conflicting demands on the design. It is therefore rare to have a single (ideal) Pareto optimal point that minimizes all objectives simultaneously. More likely is the existence of a set of Pareto optimal points which are indifferent from each other: a Pareto front.

As in SOO, finding globally optimal solutions with certainty is often an exceedingly difficult task, requiring a more or less exhaustive search of the feasible set (Custódio and Madeira 2018). Many algorithms - especially those with some convergence guarantee - are therefore designed to address the more tractable problem of finding local Pareto optimizers. Like their single-objective counterparts, these points are (only) optimal in some neighborhood and satisfy the condition of Pareto criticality (Custódio et al. 2011).

\section{Direct multisearch}

In Custódio et al. (2011), the authors introduce DMS as a MOO methodology that can be used to solve a broad spectrum of constrained problems. The term refers to a class of algorithms 
whose basic structure is modeled after SOO strategies of the directional direct search type, for instance, generating set search (Kolda et al. 2003). The characteristic feature is the sampling of the objective function on a regular pattern of points, which is formed by taking steps from the iterate along multiple specified directions. Being a direct search method, DMS does not explicitly use gradient information in the optimization process. Nonetheless, given some conditions on the pattern, the method globally converges to at least one Pareto critical point even if the objectives are not differentiable (Custódio et al. 2011). For the reader's convenience, we recall the procedure and terminology of a basic DMS method (with empty search step) in the remainder of this section.

The DMS algorithm describes the evolution of a list of points which forms an approximation of the Pareto optimal set. Denoting the iteration number with a subscript $k$ we write this iterate list as a matrix $\mathbf{X}_{k}$ whose columns represent the member points:

$$
\mathbf{X}_{k}=\left[\mathbf{x}_{k}^{(1)}, \ldots, \mathbf{x}_{k}^{\left(r_{k}\right)}\right] \in \mathbb{R}^{n \times r_{k}} .
$$

The iterate list contains previously evaluated, nondominated points, and will therefore gradually develop towards better representations of the true front. Associated to each individual point is a step size parameter $\delta>0$, which is kept in the vector

$$
\boldsymbol{\delta}_{k}=\left[\delta_{k}^{(1)}, \ldots, \delta_{k}^{\left(r_{k}\right)}\right]^{T} \in \mathbb{R}^{r_{k}} .
$$

The compound list $L_{k}=\left\{\left(\mathbf{X}_{k} ; \boldsymbol{\delta}_{k}\right)\right\}$ keeps track of all such pairs. The lists are initialized with at least one feasible element $\mathbf{x}_{0} \in \Omega$, where $\mathbf{f}\left(\mathbf{x}_{0}\right)<\infty$, and a step size parameter $\delta_{0}$. If there is a single initial point, for example, then $L_{0}=\left\{\left(\mathbf{x}_{0} ; \delta_{0}\right)\right\}$.

Each iteration starts with the selection of a single iterate point $\mathbf{x}_{k}$ from the list $\mathbf{X}_{k}$. This point serves as the sampling center for the active iteration. The algorithm designer or user is free to determine the selection strategy and may tailor it to emphasize certain aspects in the optimization solution. For instance, if a balanced point distribution along the Pareto front is desired, one might prefer to explore the neighborhood of points lying near gaps in the front (Custódio et al. 2011).

In the poll step, a local search is performed around the iterate point (also called poll center). A pattern of points that surrounds the poll center (the stencil) determines where the objective function is probed. The size and shape of the stencil are given by the step size parameter of the current iterate $\left(\delta_{k}\right)$ and by a set of search directions

$$
\mathbf{D}_{k}=\left[\mathbf{d}_{k}^{(1)}, \ldots, \mathbf{d}_{k}^{\left(m_{k}\right)}\right] \in \mathbb{R}^{n \times m_{k}} .
$$

The particular choice of search directions is also left as a design decision in the general DMS framework. However, it is clear that this choice may greatly influence progress rates and convergence properties; these effects will be explored in subsequent sections.

The objective function is then evaluated on the poll points

$$
\mathbf{x}_{k}+\delta_{k} \mathbf{d}_{k}^{(i)}, \quad i \in\left\{1,2, \ldots, m_{k}\right\}
$$

as long as these points fall within the feasible set. The trial list $L_{\text {trial }}$ is constructed by including the elements $\left(\mathbf{x}_{k}+\delta_{k} \mathbf{d}_{k}^{(i)} ; \delta_{k}\right)$ in $L_{k}$, filtering out all dominated elements, and then selecting a nonempty subset that includes all nondominated points originating from $L_{k}$. If $L_{\text {trial }} \neq L_{k}$, the poll is declared successful.

In directional direct search methods, the step size parameter is changed based on the iteration being successful or unsuccessful. In particular, successful iterations are followed by an upscaling of the parameter with the expansion factor $\phi_{k} \geq 1$ : 


$$
\delta_{k+1}=\phi_{k} \delta_{k}
$$

Note that $\phi_{k}=1$, i.e. no expansion, is also a possibility. Upon unsuccessful iterations, the step length is downscaled with the contraction factor $\theta_{k}$

$$
\delta_{k+1}=\theta_{k} \delta_{k}
$$

Here $0<\theta_{k}<\theta_{\max }<1$ since contraction is a strict requirement. The modification of $\delta$ should be propagated to every element of the iterate list that was involved in the poll (meaning both the poll center and any newly added point).

The step length control parameter is used to define the main convergence criterion: the algorithms typically iterate until the step length control parameter drops below some user-defined threshold $\delta_{\text {tol }}$ for all points in the iterate list. Of course, other budget constraints such as limits on the number of function evaluations or elapsed time can also be considered to trigger earlier termination.

\section{Simplex derivatives for vector-valued functions}

While direct search techniques are often designed to handle nonsmooth problems, it can still be beneficial to include local sensitivity information in the optimization process. A common approach is to use simplex derivatives to bridge the gap between derivative-free and gradient-based methods. In the single-objective setting, simplex derivatives have been used to accelerate (generalized) pattern search (Custódio and Vicente 2007) and generating set search (Frimannslund and Steihaug 2007; Dedoncker et al.|2021) and also form an integral part of implicit filtering methods (Kelley 2011). A multi-objective version of implicit filtering was recently developed by Cocchi et al. (2018).

Given a vector-valued function $\mathbf{f}: \mathbb{R}^{n} \rightarrow \mathbb{R}^{p}$, the first-order simplex derivative at a central point $\mathbf{x}$ is an approximation to the classical derivative which is obtained by constructing a linear interpolation or regression model on a set of points surrounding $\mathbf{x}$. Suppose that $\mathbf{f}$ is sampled at $m+1$ points $\mathbf{x}^{(i)}$,

$$
\mathbf{f}^{(i)}=\mathbf{f}\left(\mathbf{x}^{(i)}\right), \quad i \in\{0,1, \ldots, m\}
$$

where $\mathbf{x}^{(0)}$ represents the central point in the sample set. For this set, define the approximate simplex diameter as $\delta_{\max }=\max _{i}\left\{\left\|\mathbf{x}^{(i)}-\mathbf{x}^{(0)}\right\|\right\}$. Furthermore, define the matrices $\Delta \mathbf{X} \in \mathbb{R}^{n \times m}$ and $\boldsymbol{\Delta} \mathbf{F} \in \mathbb{R}^{p \times m}$ respectively as

$$
\begin{aligned}
& \Delta \mathbf{X}=\left[\mathbf{x}^{(1)}-\mathbf{x}^{(0)}, \ldots, \mathbf{x}^{(m)}-\mathbf{x}^{(0)}\right], \\
& \boldsymbol{\Delta} \mathbf{F}=\left[\mathbf{f}^{(1)}-\mathbf{f}^{(0)}, \ldots, \mathbf{f}^{(m)}-\mathbf{f}^{(0)}\right] .
\end{aligned}
$$

The simplex Jacobian $\mathbf{J}_{s} \mathbf{f}\left(\mathbf{x}^{(0)}\right) \in \mathbb{R}^{p \times n}$ is found as the solution to the (least-squares) system

$$
\mathbf{J}_{s} \mathbf{f}\left(\mathbf{x}^{(0)}\right) \in \arg \min _{W}\|\mathbf{W} \boldsymbol{\Delta} \mathbf{X}-\boldsymbol{\Delta} \mathbf{F}\|_{F}^{2} .
$$

The system might be underdetermined; in this case, the minimum-norm solution is taken Conn et al. 2008). The Moore-Penrose pseudoinverse is used to concisely represent both cases as

$$
\mathbf{J}_{s} \mathbf{f}\left(\mathbf{x}^{(0)}\right)=\Delta \mathbf{F}(\Delta \mathbf{X})^{\dagger}
$$


To ensure that the simplex Jacobian is an accurate approximation to the real Jacobian, the geometry of the sample set is an important factor. The following theorem gives an error bound on the simplex gradient - that is, the transpose of the simplex Jacobian - in case $\mathbf{f}$ is scalar-valued (Custódio and Vicente 2007; Conn et al. 2008).

Theorem 1 (Error bound on the gradient approximation). Let $\left\{\mathbf{x}^{(0)}, \mathbf{x}^{(1)}, \ldots, \mathbf{x}^{(m)}\right\} \subset \mathbb{R}^{n}$ be a sample set for constructing the simplex gradient $\nabla_{s} f\left(\mathbf{x}^{(0)}\right)$. Assume that $\operatorname{rank}(\boldsymbol{\Delta} \mathbf{X})=$ $\min (m, n)$. Let $\hat{\mathbf{U}}_{X} \hat{\boldsymbol{\Sigma}}_{X} \hat{\mathbf{V}}_{X}^{T}$ represent the reduced ('economy-size') singular value decomposition of $\Delta \mathbf{X}^{T} / \delta_{\max }$. Assume that $\nabla f$ is Lipschitz continuous on an open domain containing the enclosing ball $\mathcal{B}\left(\mathbf{x}^{(0)} ; \delta_{\max }\right)$ with constant $\gamma>0$.

Then the error of the simplex gradient at $\mathbf{x}^{(0)}$, as an approximation to $\nabla f\left(\mathbf{x}^{(0)}\right)$, satisfies

$$
\left\|\hat{\mathbf{V}}_{X}^{T}\left[\nabla_{s} f\left(\mathbf{x}^{(0)}\right)-\nabla f\left(\mathbf{x}^{(0)}\right)\right]\right\| \leq\left(\sqrt{m} \frac{\gamma}{2}\left\|\hat{\mathbf{\Sigma}}_{X}^{-1}\right\|\right) \delta_{\max }
$$

Corollary 1. Assume that each component of $\mathbf{f}: \mathbb{R}^{n} \rightarrow \mathbb{R}^{p}$ has a Lipschitz continuous gradient on an open domain containing the enclosing ball $\mathcal{B}\left(\mathbf{x}^{(0)} ; \delta_{\max }\right)$ with constant $\gamma>0$.

Then the error of the simplex Jacobian $\mathbf{J}_{s} \mathbf{f}\left(\mathbf{x}^{(\mathbf{0})}\right)$, as an approximation to $\mathbf{J} \mathbf{f}\left(\mathbf{x}^{(\mathbf{0})}\right)$, satisfies

$$
\left\|\hat{\mathbf{V}}_{X}^{T}\left[\mathbf{J}_{s} \mathbf{f}\left(\mathbf{x}^{(\mathbf{0})}\right)-\mathbf{J f}\left(\mathbf{x}^{(\mathbf{0})}\right)\right]^{T}\right\|_{F} \leq\left(\sqrt{p m} \frac{\gamma}{2}\left\|\hat{\boldsymbol{\Sigma}}_{X}^{-1}\right\|\right) \delta_{\max }
$$

Proof. Acknowledging that each of the $p$ rows in $\mathbf{J f}$ is the (transposed) gradient of one component function, the result follows immediately from 13 .

Note that the error can only be bounded as far as the component inside the column space of $\hat{\mathbf{V}}_{X}$ (which is that of $\boldsymbol{\Delta} \mathbf{X}$ ) is concerned, which is reasonable as the sensitivity of $\mathbf{f}$ in other directions is not revealed with this sample set. If there are $n$ linearly independent columns in $\boldsymbol{\Delta} \mathbf{X}$, then $\hat{\mathbf{V}}_{X}$ is square and $\sqrt{13}$ is a total error bound. The theorem shows that the simplex gradient error is linear in the approximate simplex diameter $\delta_{\max }$ and that the constant depends on the sample set geometry through $\left\|\hat{\Sigma}_{X}^{-1}\right\|$. Well-poised sample sets minimize this quantity by using steps of similar lengths in dissimilar directions Conn et al. (2009).

\section{Adaptive direct multisearch using simplex derivatives}

\subsection{Conceptual approach}

Only a small conceptual step separates directional sampling, as used in DMS, from the calculation of a simplex Jacobian using 12 . All the function evaluations needed to construct a Jacobian at the iterate point are an integral part of the standard DMS procedure, so the cost of additional (expensive) black-box queries is not a concern. Our goal in the present work is to accelerate the basic DMS algorithm proposed by Custódio et al. (2011), through exploiting this connection. More specifically, we use the simplex Jacobian to determine more effective search directions with a relatively small computational effort.

We take inspiration from the work of Fliege and Svaiter (2000), in which the steepest descent direction $\mathbf{g}_{d}$ for the (vector-valued) objective function $\mathbf{f}$ at design point $\mathbf{x}$ is defined as the solution to the minimax problem

$$
\min _{\hat{\mathbf{g}}} \max _{i}\left\{(\mathbf{J} \hat{\mathbf{g}})^{(i)}\right\}+\frac{1}{2} \hat{\mathbf{g}}^{T} \hat{\mathbf{g}}
$$


where $\mathbf{J} \equiv \mathbf{J f}(\mathbf{x})$. This direction minimizes the upper bound on the linearized reduction across all function components (the quadratic term is for regularization). In our approach, a simplex Jacobian approximates $\mathbf{J}$ and a slightly modified version of 15 is solved to obtain a descent stencil rather than a single direction.

Algorithm 1 provides a more detailed description of the steepest-descent direct multisearch (SD-DMS) method we propose. As an instance of DMS, the algorithm closely mirrors the outline given in Section 3. Its key feature is found in the third step, where the search directions are selected and propagated. The following parts describe this procedure in more detail.

\subsection{Adaptive selection of the search directions}

In the context of MOO, the 'effectiveness' of a search direction is also dependent on multiple criteria. Any method with a posteriori articulation of preferences has to distribute its efforts between improving the incumbent Pareto optima and creating diversity in the nondominated set.

The implication for the DMS search directions is that they should be oriented towards design areas that are (prospectively) either dominant over or indifferent to the iterate point. However, some exploratory directions should be included to cope with nonlinearity, since the simplex Jacobian only provides a linear model of the true objective function. Furthermore, the analysis of Theorem 1 shows that the overall stencil geometry should be fairly regular, lest the model become inaccurate.

We achieve a compromise between these demands by optimizing the orientation of a stencil whose geometry is specified in advance. Suppose the column vectors in the matrix $\mathbf{G} \in \mathbb{R}^{n \times m}$ represent a favorable stencil shape. The desired internal geometry can be encoded in the Gram matrix $\mathbf{M}_{\mathbf{G}} \equiv \mathbf{G}^{T} \mathbf{G}$, which determines its factors up to an orthogonal transformation. By definition,

$$
\mathbf{G}^{T} \mathbf{G}=\mathbf{G}^{T} \mathbf{Q}^{T} \mathbf{Q G}=(\mathbf{Q G})^{T}(\mathbf{Q G})
$$

for orthogonal matrices $\mathbf{Q}$.

In analogy to the steepest descent direction, we call a set of vectors $\mathbf{G}_{d} \in \mathbb{R}^{n \times m}$ the steepest descent stencil with Gram matrix $\mathbf{M}_{\mathbf{G}}$ if it solves the minimax problem

$$
\begin{array}{ll}
\min _{\hat{\mathbf{G}}} & \max _{i, j}\left\{(\mathbf{J} \hat{\mathbf{G}})^{(i, j)}\right\} \\
\text { s.t. } & \hat{\mathbf{G}}^{T} \hat{\mathbf{G}}=\mathbf{M}_{\mathbf{G} .}
\end{array}
$$

Unlike (15), the stencil subproblem is generally not very easy to solve (and the solution may not be unique). We certainly have no closed-form solutions, so a suitable iterative optimization algorithm has to be used. Unfortunately, the defining problem is not convex because the geometry is determined by a quadratic equality constraint. The numerical solution will only yield a local minimum, but we assume that the approximation to the global minimum is fairly accurate.

Considering the error estimate (14), we investigate two well-poised stencil types in the remainder of this work. In both cases we start from the steepest descent stencil with Gram matrix $\mathbf{I}$, denoted $\mathbf{Q}_{d}$ (since it must be an orthonormal basis for $\mathbb{R}^{n}$ ). The first alternative is a complete (maximal) positive basis, and the second is a minimal positive basis:

$$
\mathbf{D}=\left[\mathbf{Q}_{d},-\mathbf{Q}_{d}\right] \text { or } \mathbf{D}=\left[\mathbf{Q}_{d},-\mathbf{Q}_{d} \mathbf{1} / \sqrt{n}\right]
$$


It is not difficult to calculate exact size and geometry factors $\delta_{\max }$ and $\left\|\hat{\boldsymbol{\Sigma}}_{X}^{-1}\right\|$ for both options. In the first case, the respective values are 1 and $1 / \sqrt{2}$, in the second case, the respective values are 1 and 1 .

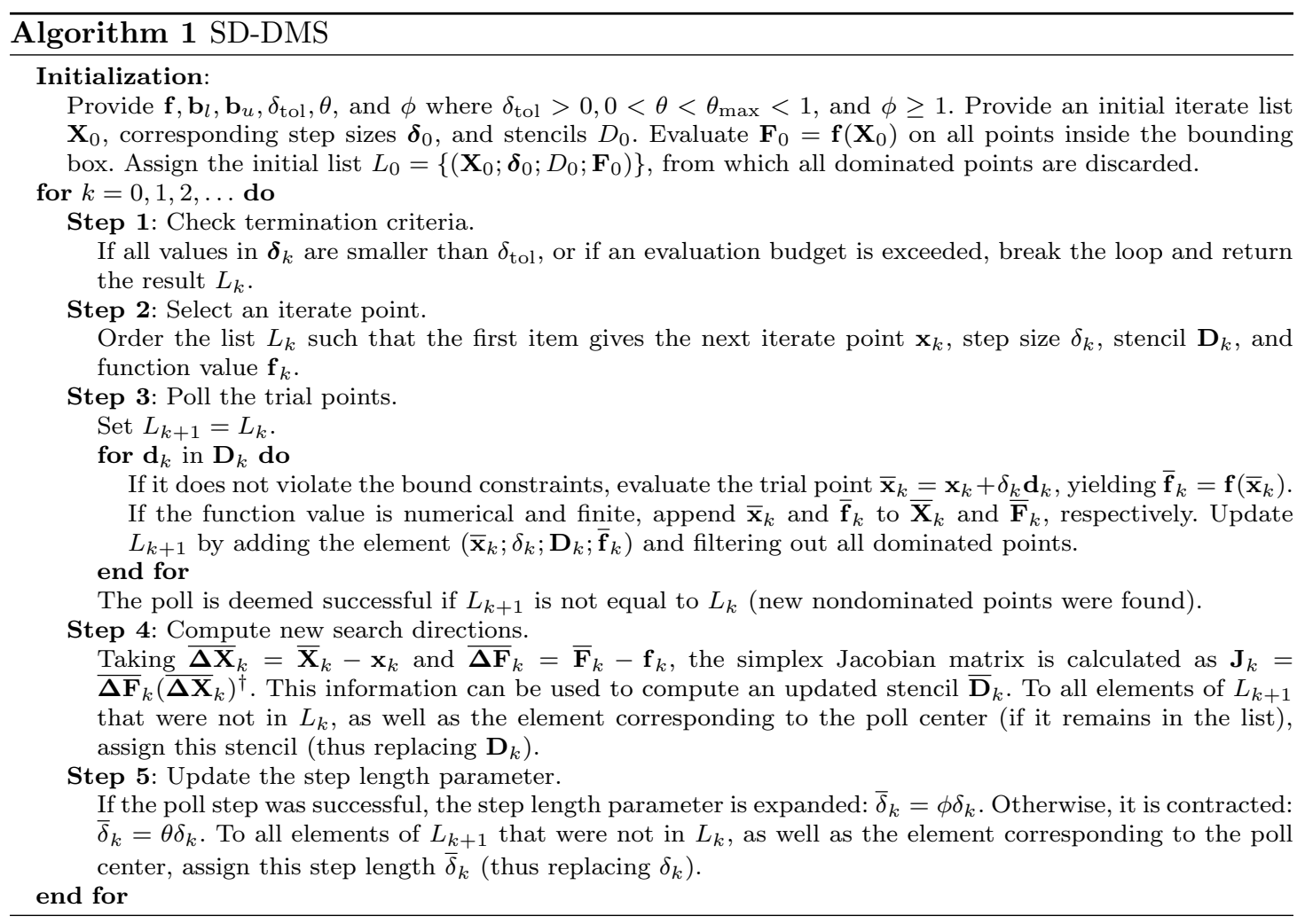

\subsection{Projection on an integer lattice}

The performance of DMS can be boosted by keeping a record of previously visited design points and their associated objective vectors (Custódio et al. 2011). This cache can be used, for example, as a lookup table for future evaluations. Apparently, the originally proposed DMS version (with fixed stencil) samples the same points often enough for this simple mechanism to drastically improve the efficiency in terms of performance per black-box function call. Clearly, the fact that this implementation uses fixed search directions plays a crucial part: this restricts the trial points to an integer lattice, increasing the probability of cache hits.

In order to benefit from caching in the SD-DMS method, we propose a 'postprocessing' step to map the optimal search directions onto a rectangular lattice. This is formalized using the operator $P: \mathbb{R}^{n} \rightarrow\{-1,0,1\}_{0}^{n}$, which matches the input to the lattice point that is closest in terms of direction:

$$
P(\mathbf{d}) \equiv \arg \max _{\mathbf{v} \in\{-1,0,1\}_{0}^{n}} \frac{\mathbf{v}^{T} \mathbf{d}}{\|\mathbf{v}\|}
$$


Herein, the notational convention

$$
\{-1,0,1\}_{0}^{n} \equiv\left\{\mathbf{w} \in \mathbb{R}_{0}^{n} \mid w^{(i)} \in\{-1,0,1\}\right\}
$$

is used to refer to the set of vectors, excluding $\mathbf{0}$, whose entries take one of the sign values $\{-1,0,1\}$.

Remark. The analysis of Custódio et al. (2011) (along with errata) reveals that DMS is globally convergent if the iterates are constrained to a rational lattice or if a sufficient decrease condition is imposed. In addition to facilitating cache usage, the projection step can therefore also play a role in the convergence of the optimization algorithm because it has the potential of restricting the iterates to a rational lattice. More specifically, our method inherits the global convergence property of DMS (Custódio et al. 2011, Theorem A.1) if (i) the search directions are projected as described above and (ii) the parameters $\theta$ and $\phi$ are finite, integer powers of a single rational number. Note that, because of the absence of an asymptotically dense set of search directions, convergence to a Pareto-Clarke stationary point is not guaranteed in the present SD-DMS implementation.

\section{Implementation aspects}

Some practical challenges and concerns arise when trying to translate the SD-DMS method to an actual computer algorithm. The subproblems that need to be solved in each iteration should be approached carefully, lest the time and memory complexity become excessive. In the following part, we discuss the main issues in this regard and offer some effective remedies.

\subsection{Subproblem reformulation for reduced complexity}

One practical challenge to solving the descent stencil subproblem is the number of design variables and constraints: assuming the stencil is a basis, these numbers scale quadratically with the dimension $n$. Since this computation is executed in every iteration, the cost may be significant. This particular difficulty may be relieved by re-framing the problem as follows.

Equation (16) shows that the geometry constraint determines the stencil up to an orthogonal transformation. This means that problem (17) can be equivalently restated as

$$
\begin{array}{ll}
\min _{\mathbf{Q}} & \max _{i, j}\left\{(\mathbf{J Q G})^{(i, j)}\right\} \\
\text { s.t. } & \mathbf{Q}^{T} \mathbf{Q}=\mathbf{I} .
\end{array}
$$

where $\mathbf{Q} \in \mathbb{R}^{n \times n}$ is orthogonal and $\mathbf{G} \in \mathbb{R}^{n \times m}$ satisfies $\mathbf{G}^{T} \mathbf{G}=\mathbf{M}_{\mathbf{G}}$. Recognizing the symmetry in this formulation, we can also interpret the problem as finding the optimal Jacobian orientation, given a fixed stencil:

$$
\begin{array}{ll}
\min _{\hat{\mathbf{J}}} & \max _{i, j}\left\{(\hat{\mathbf{J} G})^{(i, j)}\right\} \\
\text { s.t. } & \hat{\mathbf{J}} \hat{\mathbf{J}}^{T}=\mathbf{M}_{\mathbf{J}} .
\end{array}
$$

Figure 1 gives a visual representation of this changed perspective, where the design matrix $\hat{\mathbf{J}} \in \mathbb{R}^{p \times n}$ now has to conform with the Jacobian geometry $\mathbf{M}_{\mathbf{J}} \equiv \mathbf{J J}^{T}$. In general, approaching 
the problem in this manner is beneficial as long as $p<m$. Assuming $p$ is limited to a low constant - a fair assumption for most MOO problems - the number of design variables depends only linearly on $n$. The number of constraints never depends on $n$.
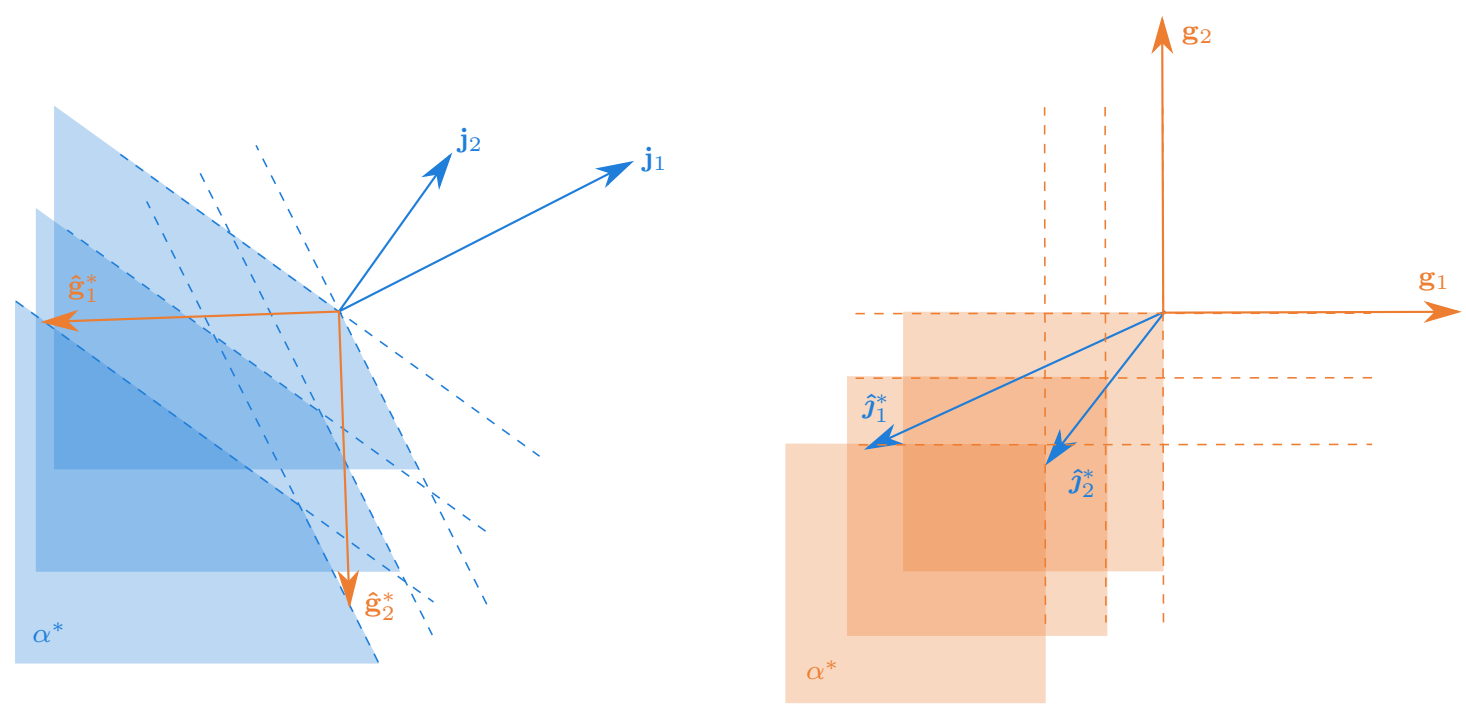

Fig. 1: The descent stencil subproblem is to minimize the maximum of the inner product between two vector sets with a certain geometry. On the left, we show the optimal $\hat{\mathbf{g}}^{*}$ for fixed $\mathbf{j}$. On the right, we show the optimal $\hat{\boldsymbol{j}}^{*}$ for fixed $\mathbf{g}$. In both cases, the colored areas represent the vectors whose inner product with the fixed set is inferior to a certain value ( $\alpha^{*}$ in the optimal case). Note that both approaches yield the same relative orientation.

After solving (21), we are left with an optimal $\hat{\mathbf{J}}^{*}$. An additional step is necessary to find the steepest descent stencil $\hat{\mathbf{G}}^{*}$. In the reformulations we have used the substitutions

$$
\mathbf{J G} \equiv \mathbf{J Q G} \equiv \hat{\mathbf{J G}} .
$$

Hence, an orthogonal transformation $\mathbf{Q}^{*}$ mapping $\mathbf{J}$ to $\hat{\mathbf{J}}^{*}$ would also take $\mathbf{G}$ to $\hat{\mathbf{G}}^{*}$ in the following way:

$$
\hat{\mathbf{J}}^{*}=\mathbf{J Q}^{*} \Longleftrightarrow \hat{\mathbf{G}}^{*}=\mathbf{Q}^{*} \mathbf{G}
$$

A valid orthogonal transformation is not difficult to find. The procedure is based on using a succession of Householder transformations that each reflect one row of $\mathbf{J}$ to the corresponding row of $\hat{\mathbf{J}}^{*}$ - see Algorithm 2 .

6.2 Finding a starting point for the steepest descent subproblem

The (original or reformulated) steepest descent problem is not convex, so providing the optimization algorithm with a good starting guess is crucial for performance. This parameter determines 


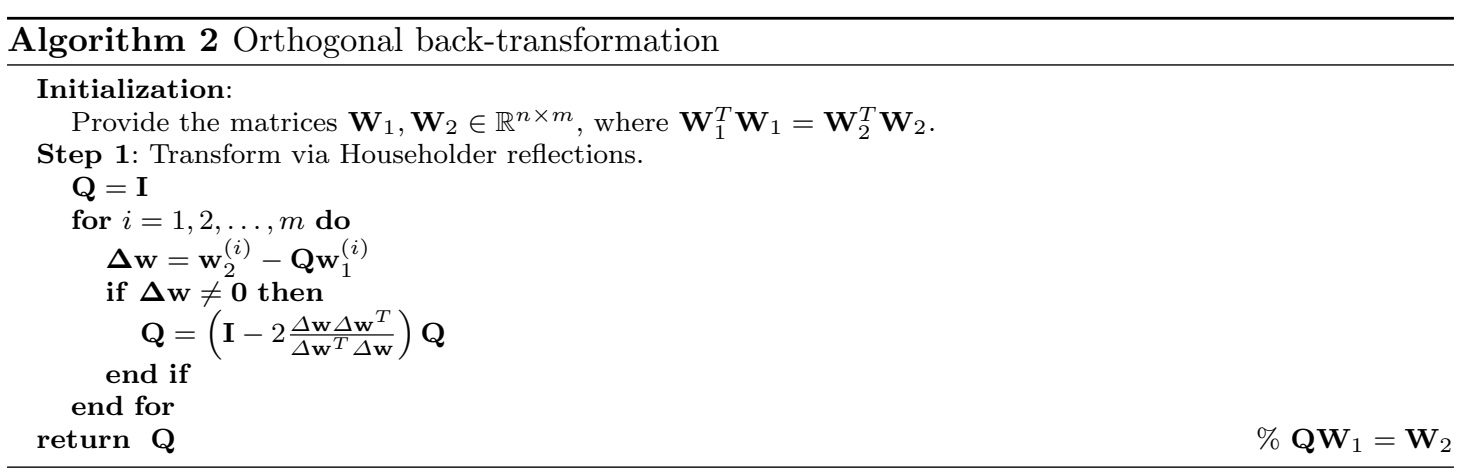

both how quickly the subproblem is solved and how well the (local) solution approximates the global minimum. The argument in the remainder of this section supposes that the original problem (17) is solved, but it also applies to the reformulation (21).

The point of defining the descent stencil is to maximize some measure of separation between the gradient vectors of the objective functions and the search directions. But even in very simple cases - see, for instance, Figure 2 - it becomes obvious that (stable) local minima also exist when the two vector sets $\mathbf{J}^{T}$ and $\hat{\mathbf{G}}$ are badly separated. For a starting point to be reliable, it should clearly avoid such situations. Therefore, we propose a selection approach that is based on an easier problem: the separation of two convex hulls. Figure 3 illustrates the approach.
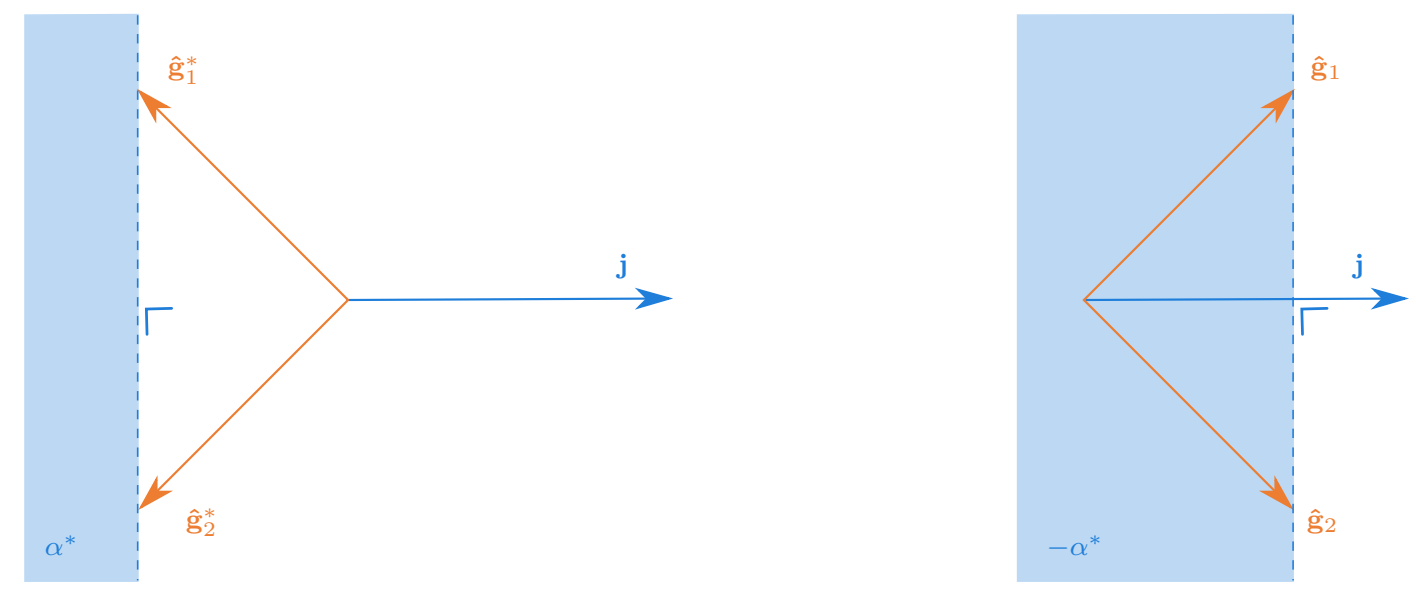

Fig. 2: The search directions $\hat{\mathrm{g}}^{*}$, shown in the left figure, form a stencil of steepest descent. However, the subproblem also has stable local minimum in this case (right figure). The colored area represents the vectors whose inner product with the gradient vector $\mathbf{j}$ is inferior to the value that labels the area.

Let conv $(\mathbf{W})$ and cone $(\mathbf{W})$ respectively denote the convex hull of and the cone generated by the matrix $\mathbf{W} \in \mathbb{R}^{n \times m}$. These are defined as the (closed, convex) subsets of $\mathbb{R}^{n}$ formed by taking, respectively, convex or positive combinations of the columns in $\mathbf{W}$ : 

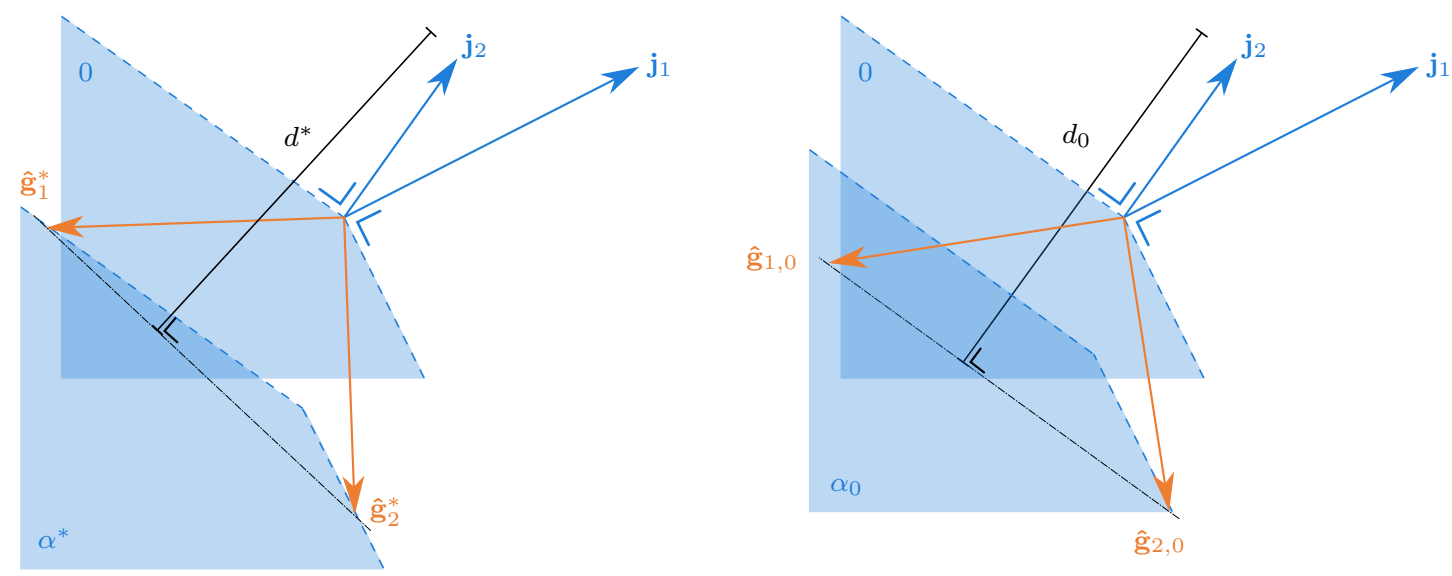

Fig. 3: The descent stencil subproblem is to minimize the maximum of the inner product between two vector sets with a certain geometry. The left figure shows the optimum of this problem. As a starting point, we choose the orientation that maximizes the distance between the convex hulls of the vector sets. This situation is shown in the right figure. In both cases, the colored areas represent the vectors whose inner product with the fixed set is inferior to a certain value. The black measurement line indicates the distance between the convex hulls. By the respective definitions, we have $\alpha^{*} \leq \alpha_{0}$ and $d^{*} \leq d_{0}$.

$$
\begin{aligned}
& \operatorname{conv}(\mathbf{W}) \equiv\left\{\mathbf{w}=\mathbf{W} \mathbf{v} \mid \mathbf{v} \geq \mathbf{0}, \mathbf{v}^{T} \mathbf{1}=1\right\} \\
& \operatorname{cone}(\mathbf{W}) \equiv\{\mathbf{w}=\mathbf{W} \mathbf{v} \mid \mathbf{v} \geq \mathbf{0}\} .
\end{aligned}
$$

For two closed sets $\mathcal{W}_{1}, \mathcal{W}_{2} \subset \mathbb{R}^{n}$, their distance is

$$
\operatorname{dist}\left(\mathcal{W}_{1}, \mathcal{W}_{2}\right) \equiv \min \left\{\left\|\mathbf{w}_{1}-\mathbf{w}_{2}\right\| \mid \mathbf{w}_{1} \in \mathcal{W}_{1}, \mathbf{w}_{2} \in \mathcal{W}_{2}\right\}
$$

Now suppose two matrices are given, whose columns each define a specific geometry. Under this constraint, we wish to find the mutual orientation that maximizes the distance between the convex hulls. Even though this is a nonconvex problem in itself, it turns out that optimizers are not that hard to find. We continue by formally proving this result - Theorem 2 - and providing a practical solution algorithm. Preliminary results can be found in Appendix A along with their proofs.

Theorem 2 (Optimal separation of two convex hulls). Given two matrices $\mathbf{W}_{1} \in \mathbb{R}^{n \times m_{1}}$ and $\mathbf{W}_{2} \in \mathbb{R}^{n \times m_{2}}$, let $\mathbf{Q} \in \mathbb{R}^{n \times n}$ be the design variable for the optimization problem

$$
\begin{array}{ll}
\max _{\mathbf{Q}} & \operatorname{dist}\left(\operatorname{conv}\left(\mathbf{Q} \mathbf{W}_{1}\right), \operatorname{conv}\left(\mathbf{W}_{2}\right)\right) \\
\text { s.t. } & \mathbf{Q}^{T} \mathbf{Q}=\mathbf{I} .
\end{array}
$$


Let $\mathbf{w}_{1}^{*}$ and $\mathbf{w}_{2}^{*}$ be the respective minimizers

$$
\mathbf{w}_{1}^{*}=\arg \min _{\mathbf{w}_{1} \in \operatorname{conv}\left(\mathbf{W}_{1}\right)}\left\|\mathbf{w}_{1}\right\| \quad \text { and } \quad \mathbf{w}_{2}^{*}=\arg \min _{\mathbf{w}_{2} \in \operatorname{conv}\left(\mathbf{w}_{2}\right)}\left\|\mathbf{w}_{2}\right\| .
$$

Supposing $\mathbf{w}_{1}^{*}, \mathbf{w}_{2}^{*} \neq \mathbf{0}$, any orthogonal $\mathbf{Q}^{*}$ that satisfies

$$
\mathbf{Q}^{*} \mathbf{w}_{1}^{*}=-\alpha \mathbf{w}_{2}^{*}
$$

with $\alpha>0$, is a solution to problem (27).

Proof. By Lemma 1, the objective satisfies

$$
\operatorname{dist}\left(\operatorname{conv}\left(\mathbf{Q} \mathbf{W}_{1}\right), \operatorname{conv}\left(\mathbf{W}_{2}\right)\right) \leq \operatorname{dist}\left(\mathbf{0}, \operatorname{conv}\left(\mathbf{Q} \mathbf{W}_{1}\right)\right)+\operatorname{dist}\left(\mathbf{0}, \operatorname{conv}\left(\mathbf{W}_{2}\right)\right) .
$$

Since the orthogonal transformation leaves distances to the origin unchanged,

$$
\operatorname{dist}\left(\mathbf{0}, \operatorname{conv}\left(\mathbf{Q} \mathbf{W}_{1}\right)\right)=\operatorname{dist}\left(\mathbf{0}, \operatorname{conv}\left(\mathbf{W}_{1}\right)\right)
$$

the upper bound is constant over all possible designs $\mathbf{Q}$. In addition, Lemma 3 assures that

$$
\begin{aligned}
\operatorname{dist}\left(\operatorname{conv}\left(\mathbf{Q}^{*} \mathbf{W}_{1}\right), \operatorname{conv}\left(\mathbf{W}_{2}\right)\right) & =\operatorname{dist}\left(\mathbf{0}, \operatorname{conv}\left(\mathbf{Q}^{*} \mathbf{W}_{1}\right)\right)+\operatorname{dist}\left(\mathbf{0}, \operatorname{conv}\left(\mathbf{W}_{2}\right)\right) \\
& =\operatorname{dist}\left(\mathbf{0}, \operatorname{conv}\left(\mathbf{W}_{1}\right)\right)+\operatorname{dist}\left(\mathbf{0}, \operatorname{conv}\left(\mathbf{W}_{2}\right)\right)
\end{aligned}
$$

because the condition 28 holds. Therefore, $\mathbf{Q}^{*}$ achieves the largest possible value of the objective, making it a solution of the separation problem.

Corollary 2. If $\mathbf{Q}^{*}$ satisfies (28), then

$$
\text { cone }\left(\mathbf{Q}^{*} \mathbf{W}_{1}\right) \cap \text { cone }\left(\mathbf{W}_{2}\right)=\{\mathbf{0}\}
$$

Proof. The generators of both cones are in their respective convex hull by definition. Since $\mathbf{Q}^{*}$ satisfies (28), and according to Lemma 2, the vector $\mathbf{w}_{2}^{*}$ therefore has a strictly positive inner product with the generators $\mathbf{W}_{2}$ and a strictly negative inner product with the generators $\mathbf{Q}^{*} \mathbf{W}_{1}$. Hence, this vector is normal to a hyperplane that strictly separates both cones at any point away from the origin.

To initialize the optimization routine for problem $(17)$, we therefore set the stencil to $\hat{\mathbf{G}}_{0}=$ $\mathbf{Q}^{*} \mathbf{G}$, where $\mathbf{Q}^{*}$ maximizes the distance between the convex hulls of $\mathbf{G}$ and $\mathbf{J}^{T}$. Algorithm 3 provides an efficient way to find $\mathbf{Q}^{*}$. Besides its low cost - basically, solving two small quadratic programs - this initialization has a number of advantages. Following Corollary 2, it is certain that the cones spanned by the initial stencil and the gradient vectors only intersect at the origin (as long as neither vector set has the origin in its convex hull). Consequently, the type of suboptimal orientation illustrated by Figure 2 is avoided. The starting point is also feasible, since it respects the geometric constraint, so a wide range of nonlinear optimization algorithms may be used. 


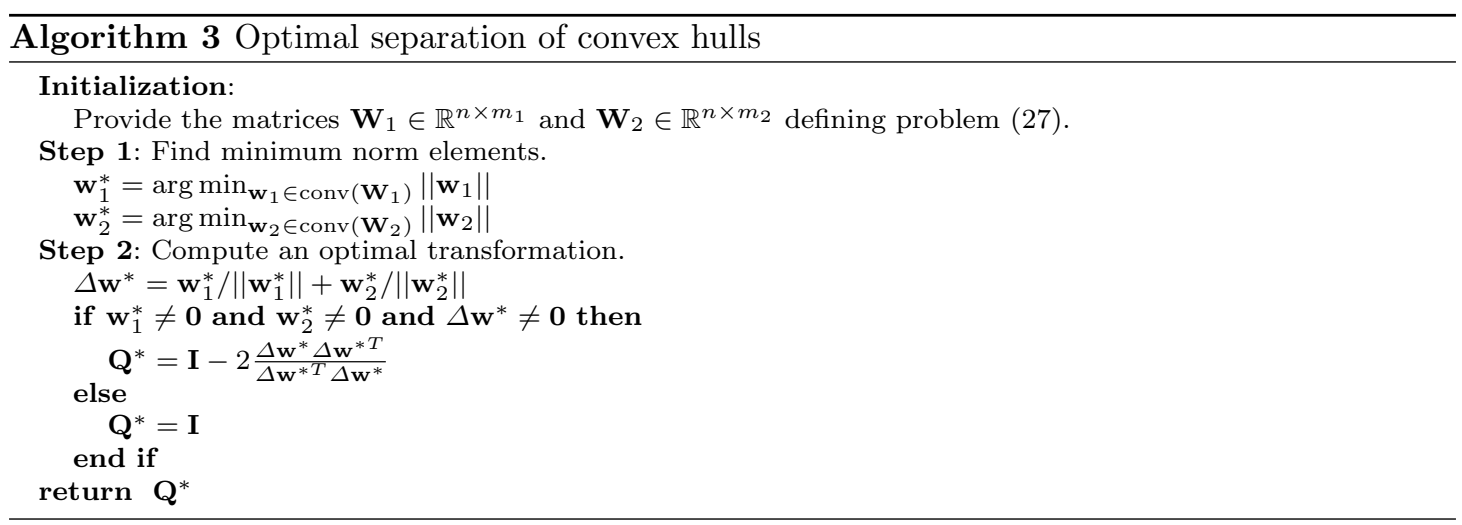

\subsection{Projection on an integer lattice}

To compute the integer approximation to a vector $\mathbf{w} \in \mathbb{R}^{n}$, we have to evaluate the function $P(\mathbf{w})$. The naive approach is to solve a combinatorial optimization problem that scales exponentially with the dimension $n$. Fortunately, the complexity can be drastically reduced by exploiting a few straightforward observations. First of all, note that the operator $P$ commutes with both permutations and sign reversals. Specifically, if $\mathbf{S}$ is a signed permutation matrix, then the identity

$$
P(\mathbf{S w})=\mathbf{S} P(\mathbf{w})
$$

holds. In other words, the negation or rearrangement of vector entries in $\mathbf{w}$ is carried over to the approximation $P(\mathbf{w})$. Now, suppose that $\mathbf{S}$ is constructed by the criterion

$$
(\mathbf{S w})^{(1)} \geq(\mathbf{S w})^{(2)} \geq \ldots \geq(\mathbf{S w})^{(n)} \geq 0 .
$$

The objective function of $P(\mathbf{S w})$ is always given by

$$
\frac{\mathbf{v}^{T} \mathbf{S w}}{\|\mathbf{v}\|}=\frac{\sum_{i=1}^{n} v^{(i)}(\mathbf{S w})^{(i)}}{\sqrt{\sum_{i=1}^{n} v^{(i)} v^{(i)}}} .
$$

In order to maximize this function, all entries of $\mathbf{v}$ should obviously be positive. The ordering of Sw implies additionally that the nonzero entries should be in the leading positions: any other permutation would decrease the inner product $\mathbf{v}^{T} \mathbf{S w}$. The approximation therefore takes the form

$$
P(\mathbf{S w})=[1, \ldots, 1,0, \ldots, 0]^{T},
$$

where the number of nonzero elements is somewhere between 1 and $n$. All that remains to be done is finding this number, which is the maximizer of

$$
\max _{j \in\{1, \ldots, n\}}\left\{\frac{\sum_{i=1}^{j}(\mathbf{S w})^{(i)}}{\sqrt{j}}\right\} .
$$

Finally, by the orthogonality of signed permutation matrices, we simply have

$$
P(\mathbf{w})=\mathbf{S}^{T} P(\mathbf{S w})
$$

for the approximation of the original vector. The entire process ultimately has negligible cost since it only involves trivial operations - ordering numbers, adding them together, and finding the maximum in a set of cardinality $n$. 


\section{Academic test problems}

We make use of the standard problem set of Custódio et al. (2011) for the first comparative tests. The set consists of 100 problems, each with 2, 3 or 4 objective functions. Design dimensions $n$ range from 1 to 30 and the feasible sets are always given in terms of bound constraints. AMPL descriptions of all problems are publicly available at the website http://www.mat.uc.pt/dms/. A budget of 20000 evaluations per problem is allowed. As suggested by the authors of the problem set, solvers are compared based on the purity, $\Gamma$ and $\Delta$ spread indicators and the results are displayed using performance profiles (Dolan and Moré 2002).

We experiment with two versions of the SD-DMS method, each of them using one of the stencils from equation (18). The version using a maximal positive basis is called SD-DMS-F (full), while the version using a minimal positive basis is called SD-DMS-R (reduced). The performance of these methods is compared to that of two state-of-the-art solvers for multi-objective problems:

- DMS (Custódio et al.|2011) (version 0.3 - http://www.mat.uc.pt/dms/);

- MOIF (Cocchi et al.|2018) (version 0.1 - http://www.iasi.cnr.it/ liuzzi/DFL/).

SD-DMS and the supporting algorithms are implemented in Matlab R2018b. Whenever the descent stencil subproblem (17) occurs, it is solved using fmincon with the sqp algorithm option. The minimum-norm elements used in Algorithm 3 are determined with the function quadprog.

As algorithmic settings are an important factor in the overall effectiveness of each particular method, we use the default options provided in the implementations of the existing solvers (see their respective documentation for more details). The DMS settings which are relevant to SDDMS are copied to our implementation. Specifically, this means that Algorithm 1 is initialized by taking $\delta_{\text {tol }}=10^{-3}, \theta=0.5$, and $\phi=1$. We also set $\mathbf{D}_{0}=[\mathbf{I},-\mathbf{I}]$ and $\delta_{0}=1$ for all points in the initial list. The caching system is identical to that of the DMS default, as is the selection mechanism for the poll center (Custódio et al. 2011, Section 6.3).
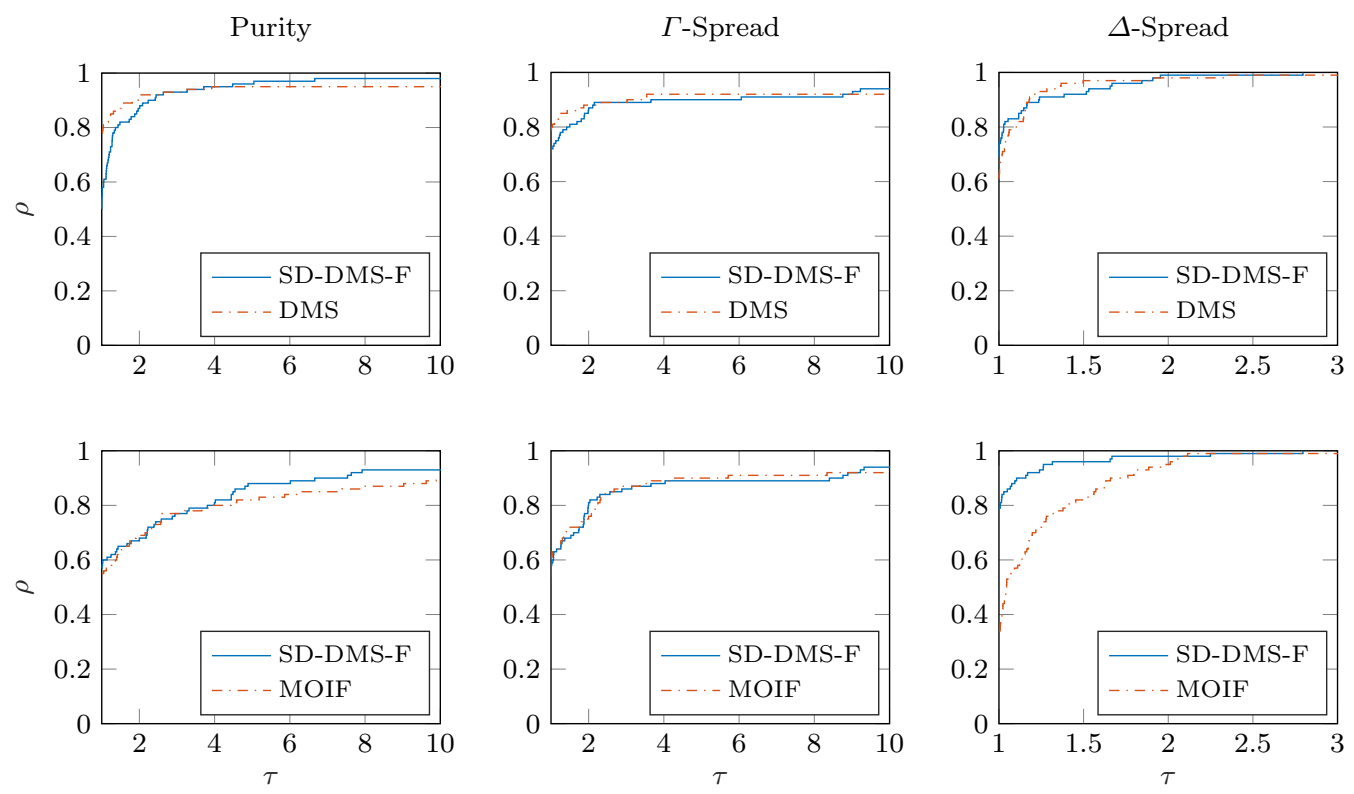

Fig. 4: Performance profiles for the benchmark set of Custódio et al. (2011). Comparison of the full (F) version of SD-DMS to state-of-the-art solvers in terms of purity, $\Gamma$ and $\Delta$ spread metrics. 

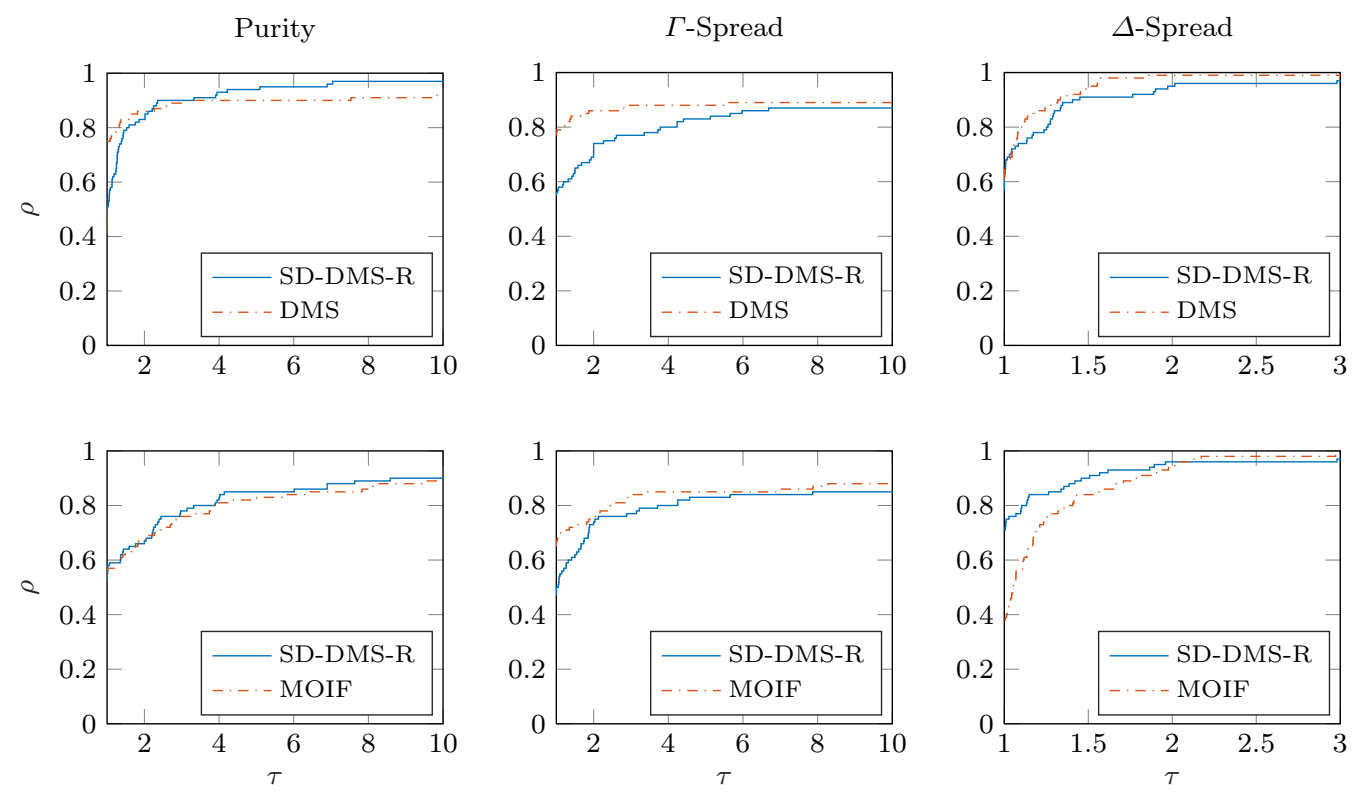

Fig. 5: Performance profiles for the benchmark set of Custódio et al. (2011). Comparison of the reduced $(\mathrm{R})$ version of SD-DMS to state-of-the-art solvers in terms of purity, $\Gamma$ and $\Delta$ spread metrics.

SD-DMS-F and SD-DMS-R are each separately compared against DMS and MOIF. The performance profiles are reported in Figures 4 and 5 . In general, the differences between the pairs are not extremely large. Especially on the top row of the first figure, where SD-DMS-F is compared to the DMS algorithm, the profiles are similar in both spread and purity. This is not greatly surprising since both methods are, in the end, very closely related: the main difference lies in stencil orientation. Since the test problems typically have uncomplicated analytical definitions, the possibility exists that the canonical directions are inherently 'preferred', so that the adaptive stencil preserves its alignment with these directions after it is projected to the integer lattice.

Inspecting the intercepts with the vertical axis on Figure 4, we observe a small advantage of the DMS method in terms of purity and $\Gamma$-spread. However, this difference is quickly nullified or reversed as the performance ratio increases. SD-DMS-F slightly improves on the purity robustness of MOIF, and also performs significantly better when considering the $\Delta$ metric. The comparisons of SD-DMS-R to DMS and MOIF (Figure 5) are mostly consistent with the trends seen in Figure 4 . The main difference lies in the spread characteristics, which seem to be slightly poorer for SDDMS-R than for SD-DMS-F.

\section{Engineering case study}

The previous section used academic test problems to assess the general performance of SD-DMS. However, performance in an academic setting does not translate directly to performance in the engineering practice. In this section, the practical use of SD-DMS is illustrated by solving a challenging engineering problem. 


\subsection{Optimization problem definition}

We consider the multidisciplinary design optimization of the mechatronic quarter-car suspension depicted in Figure 6 (Dedoncker et al. 2021). The mechanism consists of a two-dimensional system with multiple rigid bodies, configured in a four-bar linkage. By fixing the material and cross-sectional parameters, each of the bars is given a mass density of $1.56 \mathrm{~kg} / \mathrm{m}$. The leftmost bar attaches to the car body, represented by a point mass of $250 \mathrm{~kg}$, whose motion is restrained to a vertical translation. The rightmost bar is attached to the wheel hub, which has a mass of $15 \mathrm{~kg}$. The hub receives a vertical position input signal and is free to translate horizontally.

The passive and active suspension elements connect the upper left and lower right nodes of the linkage. These components are assumed to be without mass and only exert loads in the normal direction (along the connecting line). There are three elements acting in parallel. The first is a linear elastic spring, which is prestressed to support the static configuration of Figure 6 . The next component is a piecewise-linear shock damper. Its characteristic curve is an odd function of velocity given by three linear segments. The third and final element is an idealized force actuator. This component directly converts its input signal to a force in N.

The force actuator is driven by a continuous-time PI-controller. Its input is the acceleration of the body mass $\ddot{y}_{\text {out }}$ as measured by an idealized sensor. The controller has a set point of zero: we aim to keep the acceleration of the car body as small as possible (skyhook principle, see Wong $(2008))$. The internal states of the controller also equal zero at the beginning of the simulation. Finally, no output saturation is assumed to occur.

All in all, the available design parameters are as follows. Four lengths and one angle uniquely define the geometry of the linkage. The spring constant is a variable - the proper resting length

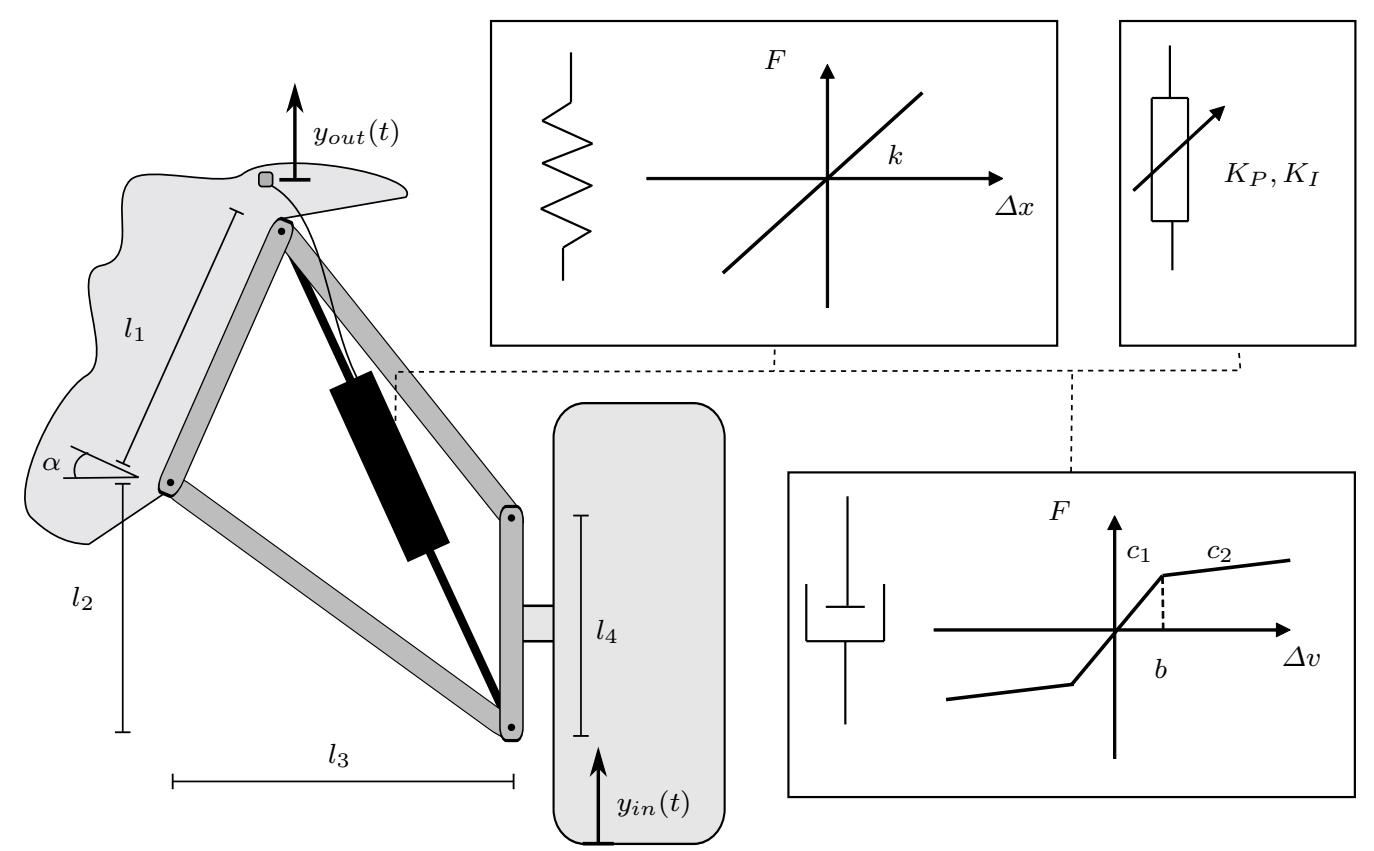

Fig. 6: Model and design parameters for the quarter-car with an active suspension consisting of a spring, nonlinear damper and PI-controlled actuator. The configuration depicts a static equilibrium, where the rightmost bar is in a vertical position. 
Table 1: Descriptions and bounds for the design variables.

\begin{tabular}{lcc}
\hline Description & Symbol & Range \\
\hline Inclination of the body link & $\alpha$ & $0^{\circ}-60^{\circ}$ \\
Length of the body link & $l_{1}$ & $0.1-0.3 \mathrm{~m}$ \\
Vertical wheel-body distance & $l_{2}$ & $0.1-0.3 \mathrm{~m}$ \\
Horizontal wheel-body distance & $l_{3}$ & $0.2-0.4 \mathrm{~m}$ \\
Length of the wheel link & $l_{4}$ & $0.2-0.4 \mathrm{~m}$ \\
Spring stiffness & $k$ & $10^{4}-10^{5} \mathrm{Nm}^{-1}$ \\
Low-speed damping coefficient & $c_{1}$ & $10^{4}-10^{5} \mathrm{Nm}^{-1} \mathrm{~s}$ \\
High-speed damping coefficient & $c_{2}$ & $5 \times 10^{3}-5 \times 10^{4} \mathrm{Nm}^{-1} \mathrm{~s}$ \\
Damping speed threshold & $b$ & $0-1 \mathrm{~ms}^{-1}$ \\
Proportional controller gain & $K_{P}$ & $0-10^{3}$ \\
Integral controller gain & $K_{I}$ & $0-10^{3}$ \\
\hline
\end{tabular}

is computed automatically for the given geometry. Next, there are three parameters governing the nonlinear damper: the slopes of the linear parts and the transition velocity. The gains of the PI-controller are the two final parameters. In total, this yields an eleven-dimensional design space, which is bounded by the values in table 1 . To prevent scaling issues, the design variables are each linearly mapped to the interval $[-1,1]$.

The goal is to find a design that optimizes dynamic performance if the wheel hub experiences a stepwise upward motion. Let $y_{\text {out }}^{(i)}, y_{\text {in }}^{(i)}$ and $F_{i n}^{(i)}$ denote displacement of the body, displacement of the wheel and road contact force sampled at time instant $t=i \times 5 \mathrm{~ms}$ after the start of the simulation. The wheel displacement is an external input which is given as

$$
y_{\text {in }}^{(i)}= \begin{cases}0 \mathrm{~m} & 0 \leq i<400 \\ 0.025 \mathrm{~m}\left[1-\cos \left(\pi \frac{i-399}{40}\right)\right] & 400 \leq i<440 \\ 0.05 \mathrm{~m} & 440 \leq i \leq 1000\end{cases}
$$

representing a smoothed step. The other quantities are computed via a (nonlinear) time-domain dynamic simulation.

The components of the objective function are given by two dimensionless scores, which are derived from basic performance indicators for ground vehicles (Wong 2008). In order to avoid bias towards either component, empirical scaling factors are introduced that bring the values to roughly the same range. The first score, which is is related to the root-mean-square value of the suspension travel (ST), is defined as

$$
\mathrm{ST}=\frac{\sqrt{\sum_{i=0}^{1000}\left(y_{\text {in }}^{(i)}-y_{\text {out }}^{(i)}\right)^{2}}}{0.2 \mathrm{~m}} .
$$

A low suspension travel is a sign that the overshoot is low and that the suspension quickly settles after disturbances, indicating responsive behavior (Wong 2008). As a second criterion, we consider the standard deviation of the road contact force (RCF) via the formula

$$
\mathrm{RCF}=\frac{\sqrt{\frac{1}{1000} \sum_{i=0}^{1000}\left(F_{i n}^{(i)}-\overline{F_{i n}}\right)^{2}}}{200 \mathrm{~N}}
$$


RCF is lower when the suspension is able to flexibly absorb shocks, minimizing dynamic tire deflections (Wong 2008). Applying these equations, we mathematically formulate the bi-objective problem as

$$
\begin{array}{ll}
\min _{\mathbf{x}} & {\left[\begin{array}{c}
\mathrm{ST}(\mathbf{x}) \\
\operatorname{RCF}(\mathbf{x})
\end{array}\right],} \\
\text { s.t. } & -\mathbf{1} \leq \mathbf{x} \leq \mathbf{1} .
\end{array}
$$

The model is implemented in the graphical environment Simulink (R2018b), so the details of its workings are unknown and simulation is automated to a large degree. The interface between the objective function and the optimization algorithm stays limited to simple evaluations. Aside from the bounds, all constraints are hidden to the user. In-depth considerations of the input parameter's internal consistency, such as geometrical compatibility, are not part of the a priori formulation. Thus, even for points inside the feasible region $\Omega$, the evaluation may not return a meaningful output within a predictable time frame. All simulations that exceed a certain time window or fail otherwise are set to return a non-numerical failure indicator.

\subsection{Methods and results}

In addition to the algorithms considered in Section 7, we use the following methods to solve the engineering problem:

- NSGA-II (Deb et al. 2002) (via the function gamultiobj in Matlab R2018b);

- BiMADS (Audet et al.2008) (via NOMAD version 3.9.1-https://www.gerad.ca/nomad/).

Given the limited budget, having many points in the initial list would only allow a few to be developed during the optimization run. Therefore, to start the optimization, the iterate lists of (SD-)DMS and MOIF are initialized with the singleton ${ }^{1} \mathbf{X}_{0}=[0,0, \ldots, 0]^{T}$. The same point is also passed as the starting design for BiMADS. The NSGA-II implementation does not offer any way to specify a starting point. Aside from the initialization, we use the default settings of the solvers and refer to their respective documentation for more details. As detailed in the previous section, SD-DMS inherits the default settings of DMS.

All six tested algorithms are given a maximal budget of 1000 black-box evaluations to generate an approximate front - for NSGA-II, this is achieved by simulating a population of size 100 over 10 generations. Given that evaluation of the objective can take up to 30 seconds, this is a reasonable limitation from a practical point of view. We only report a single run of NSGA-II, since further tests indicate that its performance has low variance across multiple runs.

Figure 7 depicts the numerical results in the form of empirical Pareto fronts for different solvers and budgets. Each of the graphs shows the suspension travel (37) on the horizontal axis and the road contact force (38) on the vertical axis. By row, each solver's best estimate is shown for 25, 50, 100, 250, 500, and 1000 evaluations. We pair the novel solvers (SD-DMS) in the right column, DMS and MOIF in the central column, and the two remaining solvers (BiMADS and NSGA-II) in the left column. Table 2 shows a hypervolume metric for each of the Pareto fronts of Figure 7. Hypervolume is an interesting multi-objective indicator because it is strictly monotonic and scales with the total dominated area. However, one should note that its value (and the relative ranking of methods) depends on an arbitrary reference point (Audet et al. 2021).

\footnotetext{
1 Strictly speaking, this is a deviation from the default settings for these algorithms.
} 


\subsection{Discussion}

Up to a budget of 100 evaluations, the four rightmost solvers generate almost the same front. This should not come as a surprise since they operate very similarly (identically in the first few steps). BiMADS and NSGA-II approach the problem from another angle, so the fronts have a different character from the start. Notably, the BiMADS approximation exhibits a 'jump' after 100 evaluations: at this point, the method identifies a new design where the suspension travel is almost zero. This behavior is physically realized by keeping the links at a dead point.

For mid-range budgets, most solvers concentrate their efforts on expanding the front towards the lower-right. After 250 evaluations, SD-DMS-R starts to diverge from the other DMS-like algorithms. The upper-left part of the front progresses more quickly towards superior designs, clearly dominating about half of the points generated by DMS, MOIF, or SD-DMS-F. NSGA-II more or less stagnates from this point onward. Around the 500-evaluation mark, we observe that BiMADS refines its approximation to resemble that of DMS (aside from the outlier found earlier). DMS and MOIF keep extending their fronts to the right.

Between 500 and 1000 evaluations, there are almost no noticeable changes for BiMADS, NSGA-II, or MOIF. Both DMS and SD-DMS-F seem to follow the same evolution as SD-DMS$\mathrm{R}$, with the adaptive method generally in a more advanced stage. Finally, SD-DMS-R itself finds the same outlier as BiMADS, and even outdoes it in the central part of the front (for RCF between 0 and 0.5 and ST between 0.5 and 1.5).

Considering these observations, we conclude that BiMADS and SD-DMS are the better solvers for the current problem. Note, however, that BiMADS is specialized to the bi-objective case, so it may be expected to perform better.

\section{Conclusion}

As the importance of numerical simulation in engineering grows, so does the need for optimization schemes that can function in tandem with (black-box) simulations. An effective optimization algorithm should be minimally affected by obstacles like numerical noise, crashes and severe discontinuities, while making the most use of the information acquired from sampling the blackbox. Furthermore, in realistic design contexts, there are often multiple goals that need to be considered simultaneously. In the present work, we have introduced an adaptive, derivativefree, multi-objective optimization method that aims to address the aforementioned challenges. Our approach is a refinement of the popular DMS method, which iteratively approximates the Pareto front by maintaining a list of nondominated points. We added an adaptive element to DMS by making the search directions evolve along with the iterate list. The selection of these

Table 2: Hypervolume metric (dominated area) per solver and budget. The reference point is $[4.622,1.049]^{T}$, which consists of the component-wise maxima over all 36 front approximations.

\begin{tabular}{lcccccc}
\hline Budget & \multicolumn{5}{c}{ Solver } \\
\hline & BiMADS & NSGA-II & DMS & MOIF & SD-DMS-F & SD-DMS-R \\
\hline 25 & 3.172 & 3.086 & 2.532 & 2.532 & 2.522 & 2.536 \\
50 & 3.193 & 3.142 & 2.903 & 2.899 & 2.915 & 2.998 \\
100 & 3.377 & 3.158 & 3.378 & 3.267 & 3.545 & 3.022 \\
250 & 3.976 & 3.168 & 3.744 & 3.723 & 3.781 & 3.850 \\
500 & 4.067 & 3.172 & 3.807 & 3.785 & 3.813 & 3.904 \\
1000 & 4.073 & 3.184 & 3.893 & 3.817 & 3.954 & 3.996 \\
\hline
\end{tabular}



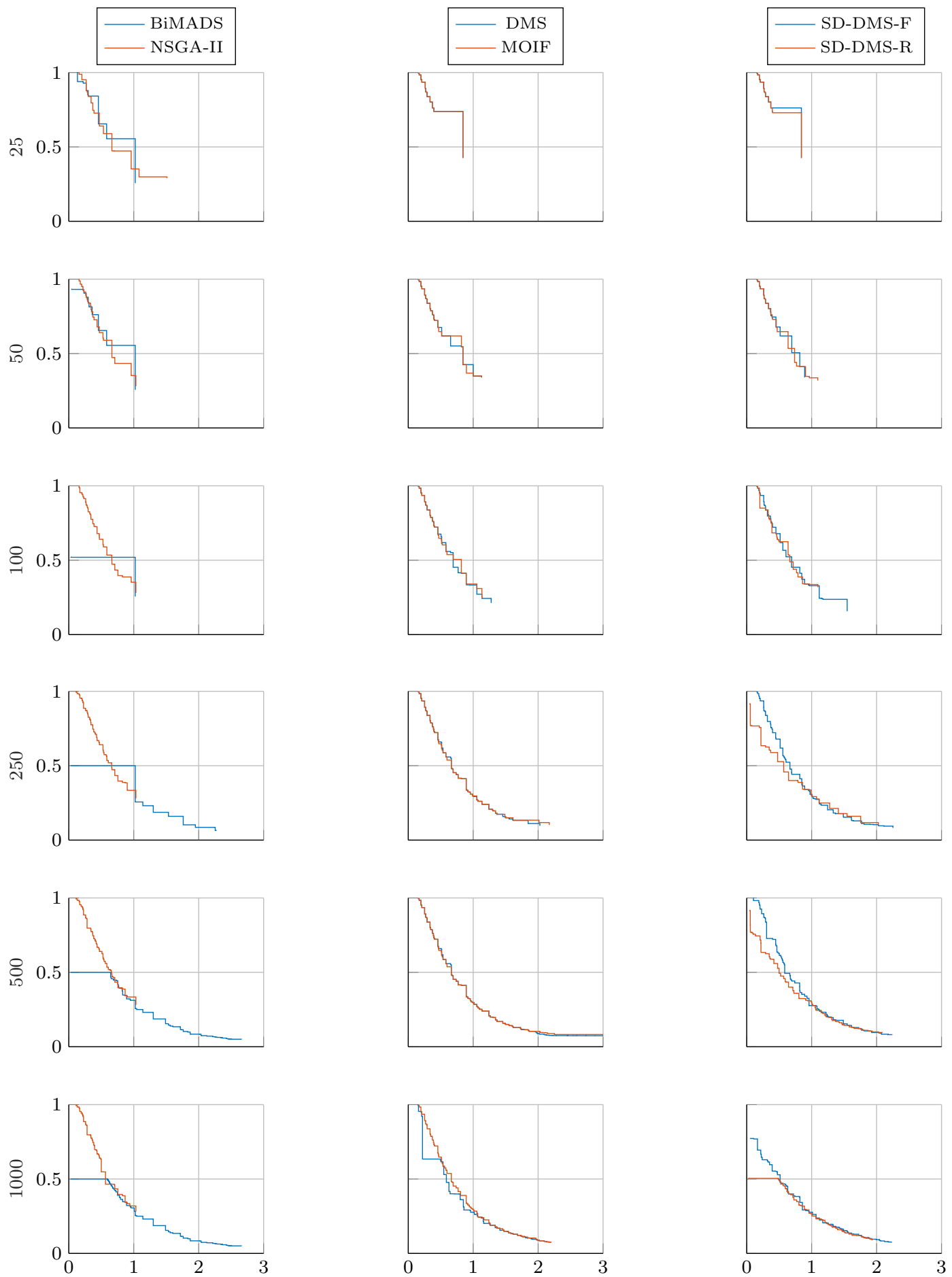

Fig. 7: Pareto front approximations for the quarter-car case. In the vertical direction, different axes correspond to the evaluation budgets shown on the far left. In the horizontal direction, different axes correspond to the solver pairs shown on top. Abscissae and ordinates correspond, respectively, to the suspension travel and road contact force indicators 37$)-(38)$. 
directions is informed by simplex derivatives. First, the concept of steepest descent (for multiobjective optimization) was generalized to sets of directions in order to derive stencils that are optimal for a linearized objective function. We then proposed some practical recommendations for solving the associated subproblems, as well as a mechanism to enable effective use of a cache. Two particular instances of the resulting steepest-descent (SD) DMS method were tested on an academic benchmark problem set and on an engineering case study, which involves bi-objective design optimization of a nonlinear quarter-car model. In the academic setting, SD-DMS-F (using a maximal positive basis) performs best in comparison to the existing DMS method. SD-DMS$\mathrm{F}$ appears similar in terms of spread, and slightly less efficient but more robust in terms of purity. In the case study, SD-DMS-R (using a minimal positive basis) is more effective, clearly outperforming several state-of-the-art solvers in terms of efficiency. The method generates better Pareto fronts with fewer function evaluations while being equally robust. Future research could focus on the behavior of SD-DMS in different situations, for instance, when the objective function has more than two components and the strong competitor BiMADS cannot be used.

\section{Acknowledgments}

This work was supported by the Research Fund KU Leuven and by Flanders Make, the strategic research center for the manufacturing industry. 


\section{A Technical results used in the proof of Theorem 2}

The first step is to recognize that the distance function has the following subadditivity property.

Lemma 1 (Subadditivity of the distance function). Let $\mathcal{W}_{1}, \mathcal{W}_{2} \subset \mathbb{R}^{n}$ be closed, and let $\mathbf{w} \in \mathbb{R}^{n}$ be an arbitrary vector. Then

$$
\operatorname{dist}\left(\mathcal{W}_{1}, \mathcal{W}_{2}\right) \leq \operatorname{dist}\left(\mathbf{w}, \mathcal{W}_{1}\right)+\operatorname{dist}\left(\mathbf{w}, \mathcal{W}_{2}\right)
$$

Proof. For arbitrary $\mathbf{w}_{1} \in \mathcal{W}_{1}, \mathbf{w}_{2} \in \mathcal{W}_{2}$, and $\mathbf{w} \in \mathbb{R}^{n}$ we have

$$
\begin{aligned}
\operatorname{dist}\left(\mathcal{W}_{1}, \mathcal{W}_{2}\right) & \leq\left\|\mathbf{w}_{1}-\mathbf{w}_{2}\right\| \\
& \leq\left\|\mathbf{w}_{1}-\mathbf{w}\right\|+\left\|\mathbf{w}_{2}-\mathbf{w}\right\|
\end{aligned}
$$

by the triangle inequality. Since this holds for any $\mathbf{w}_{1}$ and $\mathbf{w}_{2}$, it must also be the case that

$$
\begin{aligned}
\operatorname{dist}\left(\mathcal{W}_{1}, \mathcal{W}_{2}\right) & \leq \min _{\mathbf{w}_{1} \in \mathcal{W}_{1}}\left\|\mathbf{w}_{1}-\mathbf{w}\right\|+\min _{\mathbf{w}_{2} \in \mathcal{W}_{2}}\left\|\mathbf{w}_{2}-\mathbf{w}\right\| \\
& =\operatorname{dist}\left(\mathbf{w}, \mathcal{W}_{1}\right)+\operatorname{dist}\left(\mathbf{w}, \mathcal{W}_{2}\right) .
\end{aligned}
$$

The next lemma is used to show that a specific hyperplane is supporting for a convex set.

Lemma 2 (Supporting hyperplane for convex sets). Let $\mathcal{W} \subset \mathbb{R}^{n}$ be closed and convex. Let $\mathbf{w}^{*}$ be the minimizer

$$
\mathbf{w}^{*}=\arg \min _{\mathbf{w} \in \mathcal{W}}\|\mathbf{w}\| \text {. }
$$

Then, for arbitrary $\mathbf{w} \in \mathcal{W}$, the vector $\mathbf{w}^{*}$ defines a supporting hyperplane:

$$
\mathbf{w}^{T} \mathbf{w}^{*} \geq \mathbf{w}^{* T} \mathbf{w}^{*}
$$

Proof. Since the norm minimization is a convex problem, $\mathbf{w}^{*}$ exists and is a unique minimizer. By convexity, for any $\mathbf{w} \in \mathcal{W}$ and $\alpha \in[0,1]$ we have

$$
\mathbf{w}^{*}+\alpha\left(\mathbf{w}-\mathbf{w}^{*}\right) \in \mathcal{W} .
$$

The definition of $\mathbf{w}^{*}$ implies

$$
\begin{aligned}
\left\|\mathbf{w}^{*}\right\|^{2} & \leq\left\|\mathbf{w}^{*}+\alpha\left(\mathbf{w}-\mathbf{w}^{*}\right)\right\|^{2} \\
& =\left\|\mathbf{w}^{*}\right\|^{2}+2 \alpha \mathbf{w}^{* T}\left(\mathbf{w}-\mathbf{w}^{*}\right)+\alpha^{2}\left\|\mathbf{w}-\mathbf{w}^{*}\right\|^{2} .
\end{aligned}
$$

Hence

$$
0 \leq 2 \mathbf{w}^{* T}\left(\mathbf{w}-\mathbf{w}^{*}\right)+\alpha\left\|\mathbf{w}-\mathbf{w}^{*}\right\|^{2}
$$

as long as $\alpha>0$. Taking the limit $\alpha \rightarrow 0$, we find

$$
\mathbf{w}^{T} \mathbf{w}^{*} \geq \mathbf{w}^{* T} \mathbf{w}^{*}
$$

Intuitively, one might expect the optimal separation of two convex sets to occur when they are diametrically opposed across the origin. Such a situation indeed achieves an equality of the equation 40, as the next lemma demonstrates.

Lemma 3 (Distance between diametrically opposed convex sets). Let $\mathcal{W}_{1}, \mathcal{W}_{2} \subset \mathbb{R}^{n}$ be closed and convex. Let $\mathbf{w}_{1}^{*}$ and $\mathbf{w}_{2}^{*}$ be the respective minimizers

$$
\mathbf{w}_{1}^{*}=\arg \min _{\mathbf{w}_{1} \in \mathcal{W}_{1}}\left\|\mathbf{w}_{1}\right\| \quad \text { and } \mathbf{w}_{2}^{*}=\arg \min _{\mathbf{w}_{2} \in \mathcal{W}_{2}}\left\|\mathbf{w}_{2}\right\| .
$$

Suppose $\mathbf{w}_{1}^{*}, \mathbf{w}_{2}^{*} \neq \mathbf{0}$ and that $\mathbf{w}_{1}^{*}=-\alpha \mathbf{w}_{2}^{*}$ with $\alpha>0$. Then

$$
\operatorname{dist}\left(\mathcal{W}_{1}, \mathcal{W}_{2}\right)=\operatorname{dist}\left(\mathbf{0}, \mathcal{W}_{1}\right)+\operatorname{dist}\left(\mathbf{0}, \mathcal{W}_{2}\right)
$$


Proof. For arbitrary $\mathbf{w}_{1} \in \mathcal{W}_{1}, \mathbf{w}_{2} \in \mathcal{W}_{2}$ we have

$$
\begin{aligned}
\left\|\mathbf{w}_{1}^{*}\right\|\left\|\mathbf{w}_{1}-\mathbf{w}_{2}\right\| & \geq \mathbf{w}_{1}^{* T}\left(\mathbf{w}_{1}-\mathbf{w}_{2}\right) & & \text { Cauchy-Schwarz inequality } \\
& =\mathbf{w}_{1}^{* T} \mathbf{w}_{1}+\alpha \mathbf{w}_{2}^{* T} \mathbf{w}_{2} & & \mathbf{w}_{1}^{*}=-\alpha \mathbf{w}_{2}^{*} \\
& \geq \mathbf{w}_{1}^{* T} \mathbf{w}_{1}^{*}+\alpha \mathbf{w}_{2}^{* T} \mathbf{w}_{2}^{*} & & \text { Lemma } 2, \alpha>0 \\
& =\left\|\mathbf{w}_{1}^{*}\right\|^{2}+\alpha\left\|\mathbf{w}_{2}^{*}\right\|^{2} . & &
\end{aligned}
$$

Therefore,

$$
\left\|\mathbf{w}_{1}-\mathbf{w}_{2}\right\| \geq\left\|\mathbf{w}_{1}^{*}\right\|+\left\|\mathbf{w}_{2}^{*}\right\|=\left\|\mathbf{w}_{1}^{*}-\mathbf{w}_{2}^{*}\right\|,
$$

since the minimizers are aligned in opposite directions. Because the inequality holds for arbitrary $\mathbf{w}_{1}$ and $\mathbf{w}_{2}$, and because of the definition of $\mathbf{w}_{1}^{*}$ and $\mathbf{w}_{2}^{*}$, we finally obtain

$$
\operatorname{dist}\left(\mathcal{W}_{1}, \mathcal{W}_{2}\right)=\left\|\mathbf{w}_{1}^{*}-\mathbf{w}_{2}^{*}\right\|=\left\|\mathbf{w}_{1}^{*}\right\|+\left\|\mathbf{w}_{2}^{*}\right\|=\operatorname{dist}\left(\mathbf{0}, \mathcal{W}_{1}\right)+\operatorname{dist}\left(\mathbf{0}, \mathcal{W}_{2}\right)
$$

\section{References}

Audet C, Hare W (2017) Derivative-Free and Blackbox Optimization, 1st edn. Springer Series in Operations Research and Financial Engineering, Springer, Cham

Audet C, Savard G, Zghal W (2008) Multiobjective optimization through a series of single-objective formulations. SIAM Journal on Optimization 19(1):188-210

Audet C, Savard G, Zghal W (2010) A mesh adaptive direct search algorithm for multiobjective optimization. European Journal of Operational Research 204(3):545-556

Audet C, Bigeon J, Cartier D, Le Digabel S, Salomon L (2021) Performance indicators in multiobjective optimization. European Journal of Operational Research 292(2):397-422

Campana EF, Diez M, Liuzzi G, Lucidi S, Pellegrini R, Piccialli V, Rinaldi F, Serani A (2018) A multi-objective DIRECT algorithm for ship hull optimization. Computational Optimization and Applications 71(1):53-72

Cocchi G, Liuzzi G, Papini A, Sciandrone M (2018) An implicit filtering algorithm for derivative-free multiobjective optimization with box constraints. Computational Optimization and Applications 69(2):267-296

Coello Coello CA, Lechuga MS (2002) MOPSO: A proposal for multiple objective particle swarm optimization. In: Proceedings of the 2002 Congress on Evolutionary Computation, IEEE, vol 2, pp 1051-1056

Coello Coello CA, Lamont GB, van Veldhuizen DA (2007) Evolutionary algorithms for solving multi-objective problems, 2nd edn. Springer, New York

Conn AR, Scheinberg K, Vicente LN (2008) Geometry of sample sets in derivative-free optimization: Polynomial regression and underdetermined interpolation. IMA Journal of Numerical Analysis 28(4):721-748

Conn AR, Scheinberg K, Vicente LN (2009) Introduction to Derivative-Free Optimization. Society for Industrial and Applied Mathematics, Philadelphia

Custódio AL, Madeira JFA (2018) MultiGLODS: global and local multiobjective optimization using direct search. Journal of Global Optimization $72(2): 323-345$

Custódio AL, Vicente LN (2007) Using sampling and simplex derivatives in pattern search methods. SIAM Journal on Optimization 18(2):537-555

Custódio AL, Madeira JFA, Vaz AIF, Vicente LN (2011) Direct multisearch for multiobjective optimization. SIAM Journal on Optimization 21(3):1109-1140, errata at http://www.mat.uc.pt/ lnv/papers/errata-dms.pdf

Deb K, Pratap A, Agarwal S, Meyarivan T (2002) A fast and elitist multiobjective genetic algorithm: NSGA-II. IEEE Transactions on Evolutionary Computation 6(2):182-197

Dedoncker S, Desmet W, Naets F (2021) Generating set search using simplex gradients for bound-constrained black-box optimization. Computational Optimization and Applications 79(1):35-65

Dolan ED, Moré JJ (2002) Benchmarking optimization software with performance profiles. Mathematical Programming $91(2): 201-213$

Fliege J, Svaiter BF (2000) Steepest descent methods for multicriteria optimization. Mathematical Methods of Operations Research (ZOR) 51(3):479-494

Frimannslund L, Steihaug T (2007) A generating set search method using curvature information. Computational Optimization and Applications 38(1):105-121

Kelley CT (2011) Implicit filtering. Society for Industrial and Applied Mathematics, Philadelphia 
Kolda TG, Lewis RM, Torczon V (2003) Optimization by direct search: New perspectives on some classical and modern methods. SIAM Review 45(3):385-482

Liuzzi G, Lucidi S, Rinaldi F (2016) A derivative-free approach to constrained multiobjective nonsmooth optimization. SIAM Journal on Optimization 26(4):2744-2774

Marler RT, Arora JS (2004) Survey of multi-objective optimization methods for engineering. Structural and Multidisciplinary Optimization 26(6):369-395

Miettinen K (1998) Nonlinear Multiobjective Optimization. Springer US

Ryu JH, Kim S (2014) A derivative-free trust-region method for biobjective optimization. SIAM Journal on Optimization 24(1):334-362

Suppapitnarm A, Seffen KA, Parks GT, Clarkson P (2000) A simulated annealing algorithm for multiobjective optimization. Engineering Optimization 33(1):59-85

Wong JY (2008) Theory of Ground Vehicles, 4th edn. Wiley, New York

Zapotecas-Martínez S, Coello Coello CA (2016) MONSS: A multi-objective nonlinear simplex search approach. Engineering Optimization 48(1):16-38 